Ferrata Storti Foundation

\title{
Loss of plasmacytoid dendritic cell differentiation is highly predictive for post-induction measurable residual disease and inferior outcomes in acute myeloid leukemia
}

Haematologica 2018

Volume 104(7):1378-1387

\section{Correspondence:}

WENBIN XIAO

xiaow@mskcc.org

MIKHAIL ROSHAL

roshalm@mskcc.org

Received: July 25, 2018.

Accepted: December 4, 2018

Pre-published: December 6, 2018.

doi:10.3324/haematol.2018.203018

Check the online version for the most updated information on this article, online supplements, and information on authorship \& disclosures: www.haematologica.org/content/104/7/1378

(C)2019 Ferrata Storti Foundation

Material published in Haematologica is covered by copyright. All rights are reserved to the Ferrata Storti Foundation. Use of published material is allowed under the following terms and conditions:

https://creativecommons.org/licenses/by-nc/4.0/legalcode. Copies of published material are allowed for personal or internal use. Sharing published material for non-commercial purposes is subject to the following conditions:

https://creativecommons.org/licenses/by-nc/4.0/legalcode, sect. 3. Reproducing and sharing published material for commercial purposes is not allowed without permission in writing from the publisher.
Wenbin Xiao, ${ }^{1}$ Aaron D. Goldberg, ${ }^{2}$ Christopher A. Famulare, ${ }^{3}$ Sean M. Devlin, ${ }^{4}$ Nghia T. Nguyen, ${ }^{1}$ Sinnifer Sim, ${ }^{1}$ Charlene C. Kabel, ${ }^{5}$ Minal A. Patel, ${ }^{3}$ Erin M. McGovern, ${ }^{3}$ Akshar Patel, ${ }^{3}$ Jessica Schulman, ${ }^{3}$ Andrew J. Dunbar, ${ }^{2}$ Zachary D. Epstein-Peterson, ${ }^{2}$ Kamal N. Menghrajani, ${ }^{2}$ Bartlomiej M. Getta, ${ }^{2}$ Sheng F. Cai, ${ }^{2}$ Mark B. Geyer, ${ }^{2,6}$ Jacob L. Glass, ${ }^{2}$ Justin Taylor, ${ }^{2}$ Aaron D. Viny, ${ }^{2}$ Ross L. Levine,,$^{2,3,7}$ Yanming Zhang, ${ }^{8}$ Sergio A. Giralt, ${ }^{9}$ Virginia Klimek, ${ }^{2}$ Martin S. Tallman ${ }^{2}$ and Mikhail Roshal ${ }^{1}$

${ }^{1}$ Department of Pathology, Hematopathology Diagnostic Service; ${ }^{2}$ Department of Medicine, Leukemia Service; ${ }^{3}$ Center for Hematologic Malignancies; ${ }^{4}$ Epidemiology and Biostatistics; ${ }^{5} \mathrm{Clinical}$ Pharmacy; ${ }^{6}$ Center for Cell Engineering; ${ }^{7}$ Human Oncology and Pathogenesis Program; ${ }^{8}$ Department of Pathology, Cytogenetics Laboratory and ${ }^{9}$ Department of Medicine, Bone Marrow Transplant Service, Memorial Sloan Kettering Cancer Center, New York, NY, USA

\section{ABSTRACT}

M easurable residual disease is associated with inferior outcomes in patients with acute myeloid leukemia (AML). Measurable residual disease monitoring enhances risk stratification and may guide therapeutic intervention. The European LeukemiaNet working party recently came to a consensus recommendation incorporating leukemia associated immunophenotype-based different from normal approach by multi-color flow cytometry for measurable residual disease evaluation. However, the analytical approach is highly expertise-dependent and difficult to standardize. Here we demonstrate that loss of plasmacytoid dendritic cell differentiation after 7+3 induction in AML is highly specific for measurable residual disease positivity (specificity $97.4 \%$ ) in a uniformly treated patient cohort. Moreover, loss of plasmacytoid dendritic cell differentiation as determined by a blast-to-plasmacytoid dendritic cell ratio $>10$ was strongly associated with inferior overall and relapse-free survival (RFS) [Hazard ratio 2.79, 95\% confidence interval (95\% CI): 0.98-7.97; $P=0.077$ ) and 3.83 (95\%CI: 1.51-9.74; $P=0.007)$, respectively), which is similar in magnitude to measurable residual disease positivity. Importantly, measurable residual disease positive patients who reconstituted plasmacytoid dendritic cell differentiation (blast/ plasmacytoid dendritic cell ratio <10) showed a higher rate of measurable residual disease clearance at later pretransplant time points compared to patients with loss of plasmacytoid dendritic cell differentiation (blast/ plasmacytoid dendritic cell ratio $<10)$ ( 6 of $12,50 \%$ vs. 2 of $18,11 \% ; P=0.03)$. Furthermore pre-transplant plasmacytoid dendritic cell recovery was associated with superior outcome in measurable residual disease positive patients. Our study provides a novel, simple, broadly applicable, and quantitative multi-color flow cytometry approach to risk stratification in $\mathrm{AML}$ 


\section{Introduction}

Measurable residual disease (MRD) is associated with inferior outcomes in patients with acute myeloid leukemia (AML). ${ }^{1-4}$ Post-induction MRD independently predicts overall survival (OS) and relapse-free survival (RFS) ${ }^{5-10} \mathrm{MRD}$ after consolidation is also associated with a higher risk of relapse and shorter OS and RFS., ${ }^{6,11-17}$ Patients with AML who undergo hematopoietic stem cell transplant (HSCT) with any level of MRD by flow cytometry prior to HSCT are at increased risk of relapse and death. ${ }^{18-21}$

Given the prognostic significance of MRD, the 2017 European LeukemiaNet (ELN) recommends comprehensive MRD monitoring following induction and consolidation courses to assess kinetics of disease response, and then monitoring sequentially after consolidation to detect impending relapse. ${ }^{22}$ Dynamic MRD assessment for patients with AML complements baseline patient risk assessment factors, such as age, karyotype, and molecular alterations, in determining patient prognosis. More importantly, MRD status may be helpful in informing therapeutic decisions. ${ }^{6,23}$

Currently, the most commonly used methods of MRD assessment are multi-color flow cytometry (MFC) and real-time quantitative reverse transcription polymerase chain reaction (RT-qPCR) of specific mutations or gene fusions detected at diagnosis. Recently, digital PCR and next generation sequencing (NGS) have emerged as promising technologies to improve upon these methodologies. ${ }^{1,3,4}$ The major advantages of MFC are its wide applicability, assay availability, relative affordability, and rapid turnaround time, the latter facilitating prompt decisionmaking for therapeutic intervention. ${ }^{1}$ It also allows for simultaneous evaluation of expression levels of multiple antigens on the leukemic blasts, which are relevant for potential targeted immunotherapy.

Multi-color flow cytometry techniques are based on the expression of antigens that characterize diverse lineages of hematopoietic cells. AML blasts may either be defined by expression of distinct leukemia-associated immunophenotypes (LAIPs), ${ }^{16,24,25}$ or by manifesting an immunophenotypic maturation profile detected using a fixed antibody panel that is "different from normal" (DfN). ${ }^{1}$ An integrated LAIP-based DfN approach has recently been recommended by the ELN working party. ${ }^{4}$ However, this approach requires a high level of expertise for data interpretation. ${ }^{1}$ Additionally, the DfN approach has considerable variability in the equipment, reagents, data analysis methods, and reporting used in $M R D$ evaluation, confounding reproducibility and applicability outside of a limited number of expert centers with a high level of analytical expertise. ${ }^{26-28}$ Numerous analytical variables and technical obstacles are evident in the process of standardizing this MRD approach. A simpler, but nonetheless robust, approach may be beneficial in expanding risk stratification by posttreatment disease analysis outside of a few large centers.

Plasmacytoid dendritic cells (PDC) are the major natural type I interferon-producing dendritic cells that play critical roles in the immune response. ${ }^{29,30} \mathrm{PDC}$ are derived from hematopoietic stem cells and can be easily identified by flow cytometry due to high-level expression of CD123, HLA-DR and/or CD303/BDCA2 in the context of lack of lineage markers, low side scatter, and moderate expression of CD $45 .{ }^{31}$ In healthy subjects, $\mathrm{PDC}$ are $<1 \%$ of total nucleated cells in both marrow and blood, but are nonetheless present in a relatively narrow and reproducible range of proportions. ${ }^{32-34}$ Loss of PDC is observed in germline GATA-2 deficiency and increased PDC have been reported in chronic myelomonocytic leukemia. ${ }^{35-39}$ Very few studies have attempted to characterize PDC in AML and the results are inconclusive due to a small number of patients. ${ }^{32,33}$ One study suggests slightly higher levels of peripheral blood PDC at time of diagnosis in patients with FLT3-ITD ${ }^{+}$(vs. FLT3-ITD $)$AML. ${ }^{33}$ We observed a dramatic loss of PDC in most AML patients at diagnosis and investigated if the loss of PDC could be used as surrogate marker for disease persistence and risk stratification. Here we report a simple, objective, widely applicable and quantitative MFC approach using quantitation of PDC and blasts. We show that blast/PDC ratio not only correlates with MRD status but also predicts MRD clearance and outcomes in AML.

\section{Methods}

\section{Patients}

A cohort of 163 adult patients with a confirmed diagnosis of AML who underwent induction therapy with "7+3" at Memorial Sloan Kettering Cancer Center (MSKCC) between April 2014 and September 2017 was initially included. Of these, 27 had no postinduction follow-up flow cytometric data within the time frame of days 28 to 60 post induction at MSKCC and were excluded from further analysis. Post induction, 34 of $136(25 \%)$ patients had $>20 \%$ blasts and 33 of $136(24 \%)$ patients $>5 \%$ but $<20 \%$ blasts (named "residual AML"); 69 of $136(51 \%)$ patients achieved morphological remission with $<5 \%$ blasts in the marrow: 36 were MRD-negative ("MRD-neg.") and $33 \mathrm{MRD}$-positive ("MRDpos."). Complete remission (CR), CR with incomplete hematologic recovery (CRi), and morphological leukemia-free state (MLFS) were defined by the ELN criteria. ${ }^{22}$ As a control, we analyzed MFC data from two cohorts of patients (age $>18$ years old) who underwent bone marrow evaluation for mild cytopenia (absolute neutrophil counts $>1$ but $<1.5 \times 10^{9} / \mathrm{L}$, hemoglobin $>9$ but $<12$ $\mathrm{g} / \mathrm{dL}$, or platelets $>90$ but $<150 \times 10^{9} / \mathrm{L}$ ): 20 patients had a history of non-hematologic solid malignancy and another 11 patients had no history of malignancy, but had cytopenias attributable to other etiologies, such as autoimmune diseases or aplastic anemia. Morphological, cytogenetic and molecular (by a NGS platform panel composed of 28 genes frequently mutated in $\mathrm{AML}^{40}$ ) studies in the bone marrow were negative for myeloid neoplasm or clonal hematopoiesis in these control subjects. This study was approved by the Institutional Review Board of MSKCC.

\section{Measurable residual disease detection and plasmacytoid dendritic cell quantitation by flow cytometry}

Multi-color flow cytometry was performed on bone marrow aspirates at diagnosis and/or relapse and prior to induction chemotherapy. ${ }^{41}$ Briefly, up to 1.5 million cells from freshly drawn bone marrow aspirate were stained with 3-4 10-color panels (Online Supplementary Table S1), washed, and acquired on FACS Canto-10 cytometer (BD Biosciences, San Jose, CA, USA). The results were analyzed with custom Woodlist software (a generous gift from BL Wood, University of Washington, USA). An abnormal population was identified by visual assessment of populations with antigen expression that was 'different-from-normal', as described in prior publications. ${ }^{9,41}$ The assay is able to detect abnormal populations to a sensitivity of approximately 1 in 1000 
events provided at least 100,000 events are acquired in each of the three panels used. MRD detected using MFC was defined by the presence of any abnormal bone marrow blast population meeting the above criteria. ${ }^{41}$

Plasmacytoid dendritic cells were identified by low side scatter, moderate CD45, bright CD123 and HLA-DR expression, and lack of expression of CD11b, CD13, CD14, CD34, CD64, CD56, CD3, or CD19. The expression of CD303/BDCA2, TdT, and TCL-1 was examined in six selected cases with identical quantitation. Blasts were identified by low side scatter, dim CD45, positive CD34 and/or positive CD117 expression, or presence of immature monocytic forms (bright for CD33, positive for CD64 and HLADR with dim to negative expression of CD14 and/or CD11b). PDCs and blasts were quantified as the percent of total white blood cells (WBC) that is defined by CD45 positivity.

\section{Statistical analysis}

A Wilcoxon rank-sum test compared the PDC number and the blast/PDC ratio across the cohorts of patients with AML (>20\%), AML with residual disease, MRD positive disease, MRD negative disease, and non-AML controls. Cumulative incidence functions and Gray's test were used to estimate and compare the incidence of relapse by MRD status. Death in the absence of relapse was considered a competing risk. Kaplan-Meier survival curves and proportional hazards regression were used to estimate and compare overall survival and relapse-free survival by MRD status and by whether the blast/PDC ratio was $>10$. No adjustments were made for multiple comparisons. Analyses were conducted by GraphPad Prism and the R statistical language.

\section{Results}

Plasmacytoid dendritic cell proportion in control subjects

Previous studies showed that PDC were $<1 \%$ in the marrow from healthy controls..$^{32}$ In order to study the range of $\mathrm{PDC}$ in patients with cytopenia but with no marrow-based disease, we chose two cohorts of patients: one with solid tumors who developed cytopenia at least six months after completing chemotherapy, and the other with no history of malignancies. These subjects, although mildly cytopenic, had no morphological, cytogenetic or molecular evidence of myeloid neoplasm or metastatic malignancy. PDC were quantified as the percent of total WBC by flow cytometry based on low side scatter, moderate CD45, bright CD123 and HLA-DR expression (Figure $1 \mathrm{~A}$ and $\mathrm{B}$ ). The identity of the PDC was further confirmed by the expression of $\mathrm{CD} 303$ in six cases. CD303 inclusion did not alter PDC quantitation (Online Supplementary Figure S1 and data not shown). We found the PDC proportion had a relatively narrow distribution with a median of $0.23 \%$ [interquartile range (IOR): $0.16 \%$ $0.36 \%$ ] (Figure 2A). In order to evaluate the interobserver variability of the measurement of $\mathrm{PDC}$ and blast proportions, three observers (WX, SS, NN) independently quantified PDC and blast proportions for ten patients. The interclass correlation coefficient (ICC) agreement was high for both PDC and blasts (0.98 and 0.99, respectively).

\section{Marked reduction of plasmacytoid dendritic cell proportion in acute myeloid leukemia patients}

We observed a markedly decreased proportion of PDC in most patients with AML ( $>20 \%$ blasts), with a median of $0.016 \%$ (IOR: $0.0019 \%-0.071 \%$ ), more than 10 -fold lower than observed in controls (Figure 1C and D and Figure 2). There was no significant difference in PDC proportions between newly diagnosed AML and persistent AML with $>20 \%$ blasts (data not shown). Although a mild recovery of PDC proportion was observed in the patients with residual AML $(>5 \%<20$ blasts $)$ after induction chemotherapy, the median of PDC proportion was
A
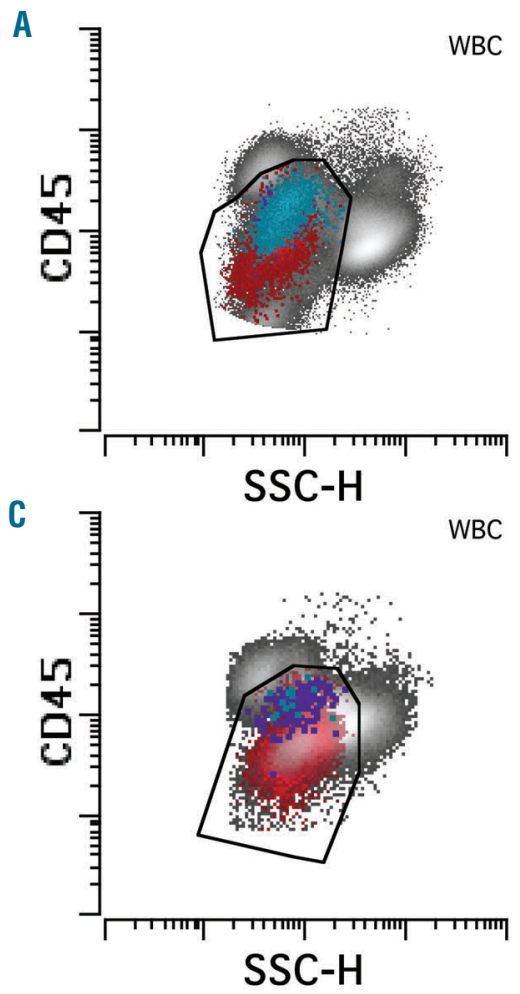
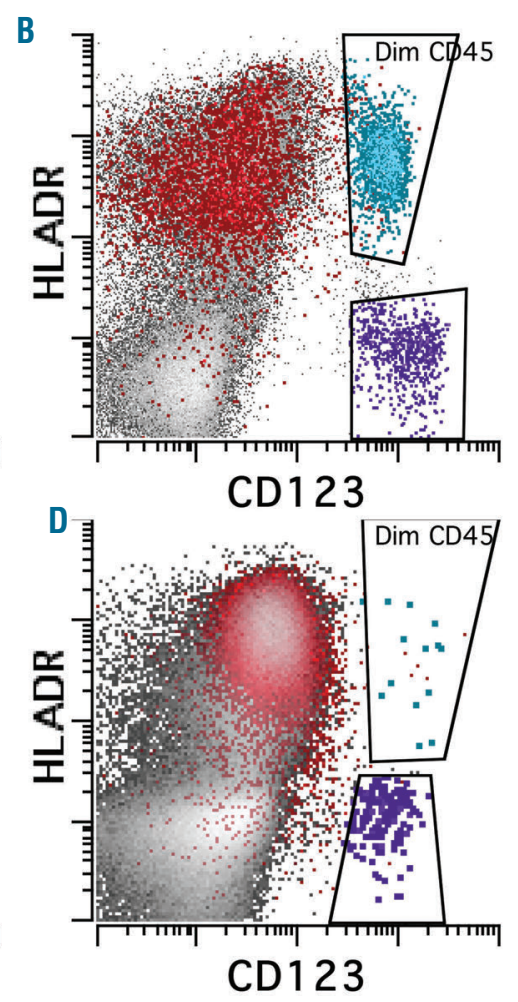

Figure 1. Loss of plasmacytoid dendritic cell (PDC) differentiation in acute myeloid leukemia (AML). PDC from a control subject ( $A$ and $B$ ) reside in the CD45 dim/low side scatter gate between blasts and monocytes, overlapping with basophils (A). PDC express high levels of CD123 and HLA-DR and can be easily separated from blasts and basophils (B). In AML with morphological disease ( $\geq 20 \%$ blasts) (C and D), PDC are markedly reduced. Red: CD34 positive blasts; blue: PDC; purple: basophils. 
0.044\% (IOR: $0.0073 \%-0.12 \%$ ), still 5-fold lower than that in controls. Five of $136 \mathrm{AML}$ patients (all had $>20 \%$ blasts in the marrow) showed an increase in PDC proportion $>1 \%$ : four newly diagnosed and one persistent AML (Figure 2A).

Increased blast to plasmacytoid dendritic cell ratio in patients with morphological remission is associated with measurable residual disease positivity

We next examined the PDC proportion in patients who achieved morphological remission (MLFS, CRi, CR by ELN criteria ${ }^{22}$ ) post induction. While there was no difference between the MRD-negative (neg) and control groups [median $0.31 \%$ (IOR: $0.17 \%-0.49 \%$ ) vs. $0.28 \%$ (IOR: $0.17 \%-0.34 \%)]$, the MRD-positive (pos) group had significantly reduced PDC proportion compared to the control (median $0.074 \%$, IOR: $0.022 \%-0.33 \%$; Wilcoxon rank sum, $P=0.019$ ) (Figure 2A). Although significant differences were observed, there was still a large overlap of PDC proportions among the groups. In an attempt to achieve better separation and to eliminate possible effects of hemodilution, we calculated the ratio of blast/PDC by using the proportions of blasts and PDC out of total WBC as quantitated by flow cytometry (Figure $2 \mathrm{~B})$. The IOR of the ratio was 341-17175 for AML, 37-385 for residual AML, 2.9-78 for MRD-pos, 2.0-3.2 for MRD-neg, and 1.12.3 for control. By using a cut-off threshold of the blast/PDC ratio of 10, all patients in the groups of AML ( $>20 \%$ blasts) and residual AML $(>5 \%<20 \%$ blasts) had a ratio of $>10$ and all controls had a ratio $<10$. Only one out of 36 patients in the MRD-neg group had a ratio $>10$. In the MRD-pos group, 19 out of $33(58 \%)$ patients had a ratio of $>10$. Therefore, a ratio of $>10$, while moderately sensitive, is highly specific for positive MRD status after induction. Overall, the area under the ROC curve was 0.79 and a threshold of 10 for the ratio had a corresponding specificity of $97.4 \%$ and a sensitivity of $58 \%$ for predicting MRD positivity status (Online Supplementary Figure S2). There was a modest positive correlation between the blast/PDC ratio and the level of MRD: rho=0.543 using Spearman rank correlation.

Both measurable residual disease positivity and blast/plasmacytoid dendritic cell ratio $>10$ are strongly associated with inferior outcomes after induction

Among the 69 patients who achieved morphological remission (CR, CRi, and MLFS), 36 (52\%) were MRD-neg and $33(48 \%)$ MRD-pos as evaluated by the MFC DfN approach. As expected, the MRD-neg group showed younger age at presentation (median 52 vs. 62 years; unpaired $t$-test, $P=0.0013$ ), and more patients with favorable CG (8 of 34 vs. 0 of 30; Fisher exact test, $P=0.005$; two MRD neg and three MRD pos patients had no CG results) and favorable ELN risk than MRD-pos group (18 of 36, $50 \%$ vs. 4 of $33,12 \%$; Fisher exact test, $P=0.0008)$. After induction therapy, there was a trend in more MRD-neg patients achieving CR (vs. CRi and MLFS) compared to MRD-pos patients (32 of 36, 89\% vs. 24 of 33, 72\%; Fisher exact test, $P=0.1)$. Although the majority of patients in both groups received consolidation therapy and HSCT, the 2 -year cumulative incidence of relapse was lower in the
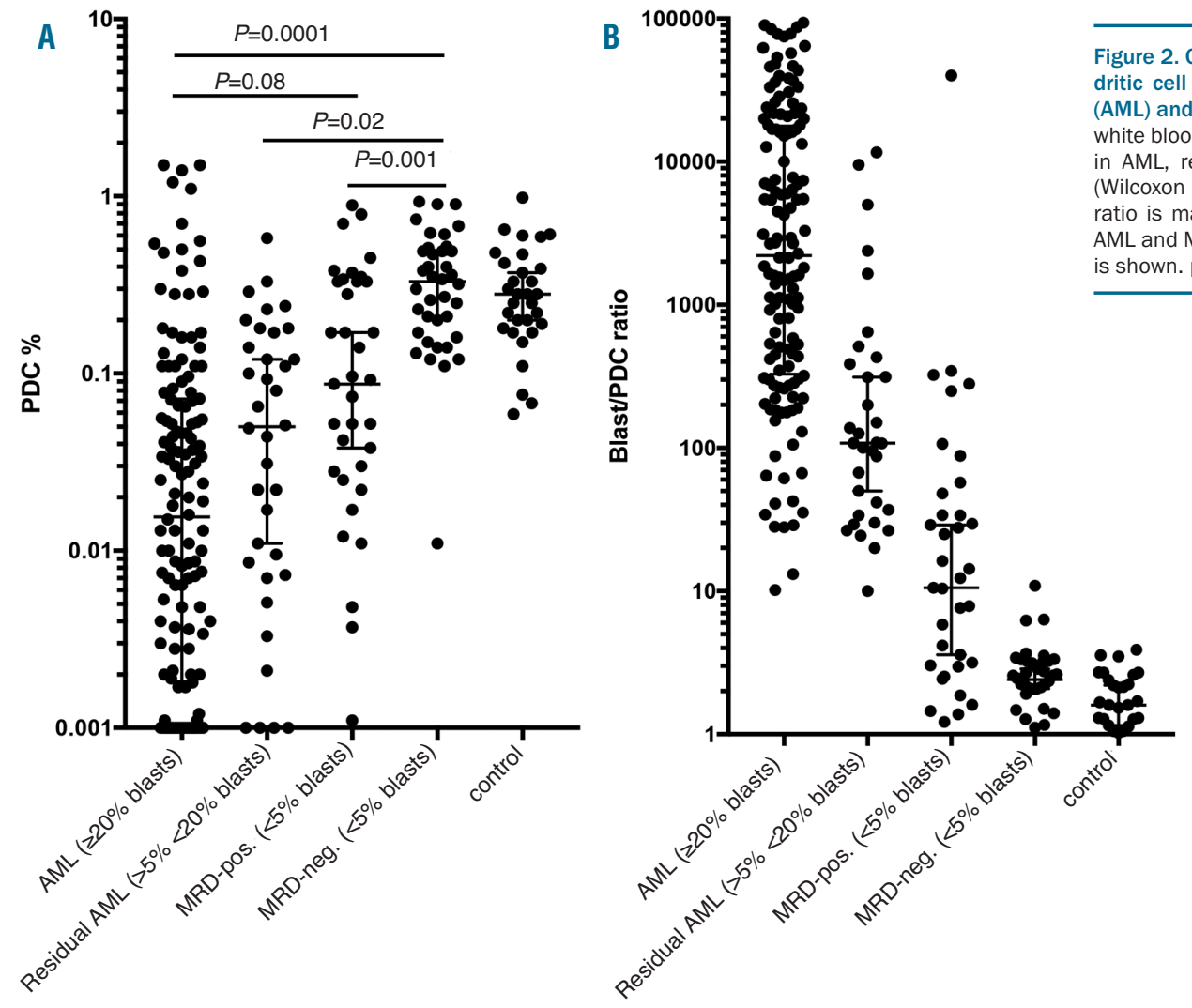
W. Ciao et al.

MRD-neg group compared to the MRD-pos group [10\% (95\% CI: $2-24 \%$ ) vs. $37 \%$ (95\% CI: $18-56 \%$ ); $P=0.014]$.

We then examined the association between post inductron MRD and survival. Consistent with published studiss, ${ }^{5-10}$ we confirmed that post induction MRD positivity was significantly associated with inferior OS and RFS in our study cohort [OS, HR 3.81 (95\% CI: 1.18-12.25), $P=0.017$; RES, HR 3.98 (95\%CI: 1.4-11.31), $P=0.007]$ (Figure $3 \mathrm{~A}$ and $\mathrm{C}$ and Online Supplementary Table S2). Given the strong association between $\mathrm{MRD}$ and blast/PDC ratio, we decided to evaluate if the blast/PDC ratio is also associated with inferior survival. Indeed, blast/PDC ratio $>10$ was also associated with inferior OS and RFS [OS, HR 2.79 (95\%CI: 0.98-7.97), $P=0.077$; RES, HR 3.83 (95\% CI: 1.51-9.74); $P=0.007$, which is similar in magnitude to MRD positivity (Figure $3 \mathrm{~B}$ and $\mathrm{D}$ and Online Supplementary Table S2). In sensitivity analysis, we also investigated the blast/PDC ratio as a continuous predictor and it remained significantly associated with OS and RFS

A

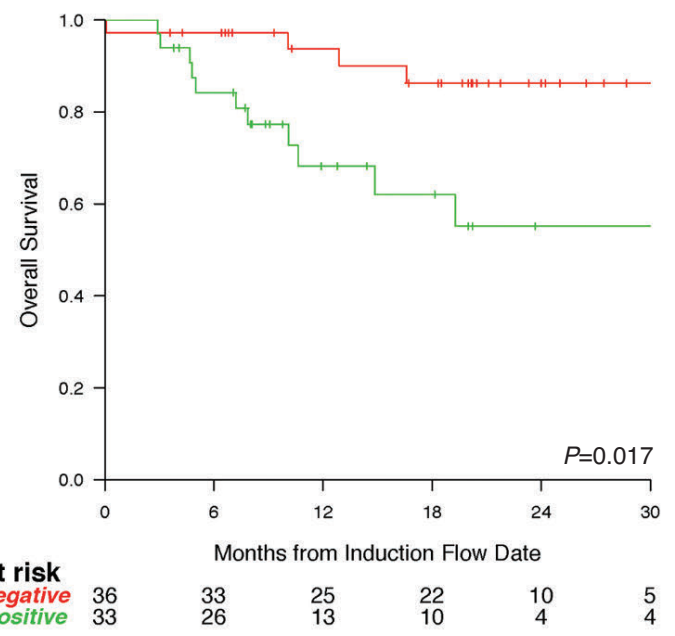

C

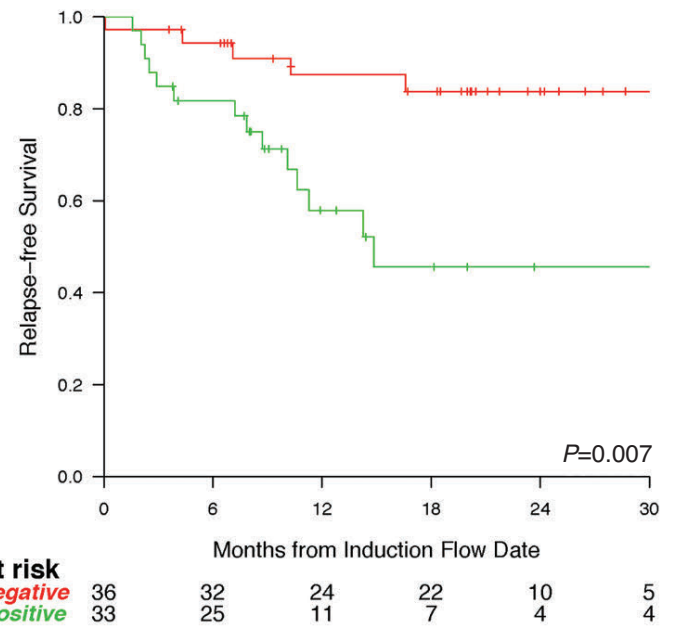

[OS HR 1.32 (95\%CI: 1.08-1.61), $P=0.006$; RES, HR 1.38 (95\%CI: 1.16-1.65), $P<0.001$; based on a log-transformatimon of the ratio] (Online Supplementary Table S2).

We next focused on patients achieving full CR (excluding CPi and MLFS). Post induction MRD positivity was significantly associated with inferior RFS and OS [RFS, HR 4.39 (95\%CI: 1.44-13.37), $P=0.009$; OS, HR 3.56 (95\%CI: 0.99-12.73), $P=0.051$ ] (Online Supplementary Table S2). Blast/PDC $>10$ was also associated with inferior RFS of similar magnitude [HR 4.93 (95\%CI: 1.71-14.2); $P=0.003$ ].

In order to examine the outcome prediction of individwal patients based on MRD and/or blast/PDC ratio, Cestatistics were performed. ${ }^{42}$ The estimated C-index using the inverse probability censoring weight for PFS was 0.80 for $\mathrm{MRD}$ positivity and 0.79 for the blast/PDC ratio $>10$. For OS, the C-index was 0.79 for MRD positivity and 0.73 for the blast/PDC ratio $>10$. Therefore, while the C-index was modestly higher for MRD, both markers show a high degree of discrimination for survival.

B

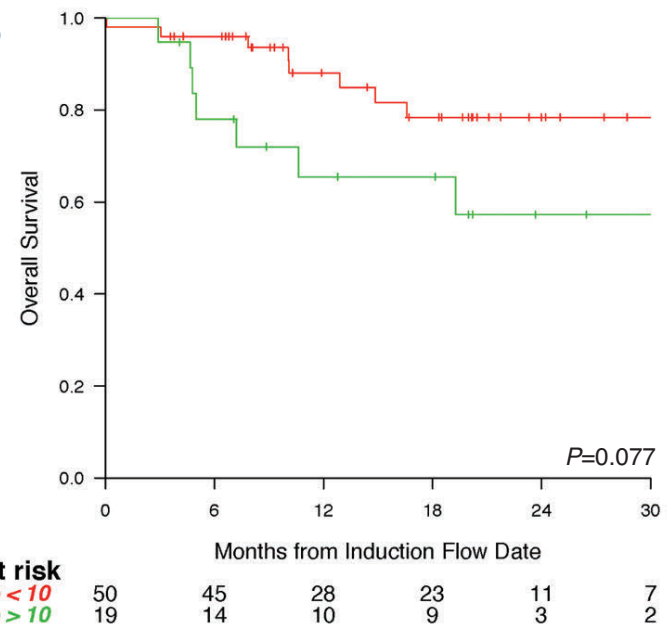

D

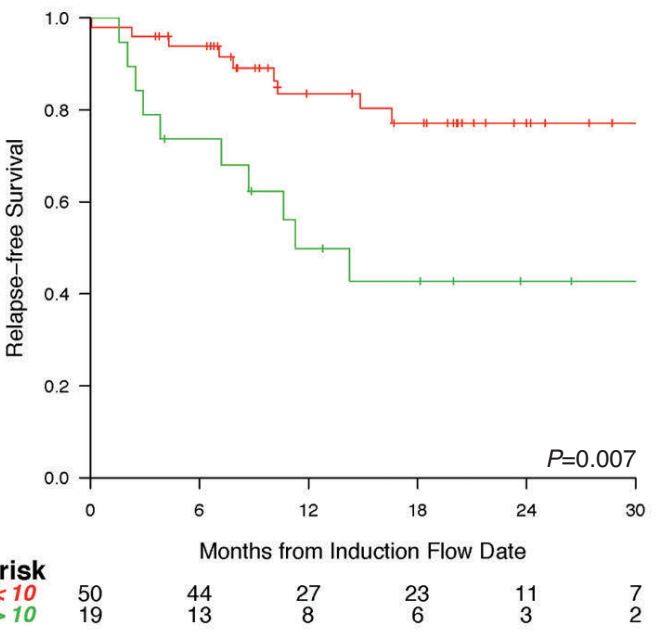

Figure 3. Kaplan-Meier survival analysis based on post-induction measurable residual disease (MRD) status and blast/plasmacytoid dendritic cell (PDC) ratio. (A) Overall survival (OS) of MRD-neg and MRD-pos groups. (B) Relapse-free survival (RFS) of MRD-neg and MRD-pos groups. (C) OS of blast/PDC ratio <10 and $>10$ groups. (D) RFS of blast/PDC ratio $<10$ and $>10$ groups. neg: negative; pos: positive.

1382

haematological | 2019; 104(7) 
In measurable residual disease-positive patients blast/plasmacytoid dendritic cell ratio $<10$ is associated with a higher measurable residual disease clearance rate

Given similar predictive values of blast/PDC ratio, and MRD despite moderate sensitivity of the blast/PDC ratio for MFC-defined MRD positivity, we examined whether blast/PDC ratio can further risk-stratify MRD-pos patients. The MRD-pos group was, therefore, subdivided based on the blast/PDC ratio: $15(45 \%)$ had a ratio $<10$ and $18(55 \%)$ a ratio $>10$. There were no differences in clinical characteristics including World Health Organization (WHO) classification, CG risk, ELN risk, and the rate of achieving CR between these two groups (Table 1). Only 8 of $15(53 \%)$ of the MRD-pos patients with
blast/PDC ratio $<10$ received consolidation therapy while all the MRD-pos patients with blast/PDC ratio $>10$ did (Fisher exact test, $P=0.003$ ). Eleven of 15 of the MRD-pos patients with blast/PDC ratio $<10$ and 14 of 18 of the MRD-pos patients with blast/PDC ratio $>10$ had received HSCT. The proportion of relapse/death in the patients with blast/PDC ratio $<10$ was 2.5 times lower approaching statistical significance (3 of 15, 20\% vs. 10 of $18,56 \%$; Fisher exact test, $P=0.07)$. Although there was no difference in OS, a trend of better RFS was also observed in patients with blast/PDC ratio $<10$ (Online Supplementary Figure S3). Additionally, estimated 18-month RFS of MRDpos patients with blast/PDC ratio $<10$ was $0.53(95 \% \mathrm{CI}$ : $0.22-0.99$ ), a little higher than that of MRD-pos patients with blast/PDC ratio $>10$ (0.39, 95\%CI: 0.21-0.73) (Online

Table 1. Clinical characteristics of patients achieving complete remission after induction.

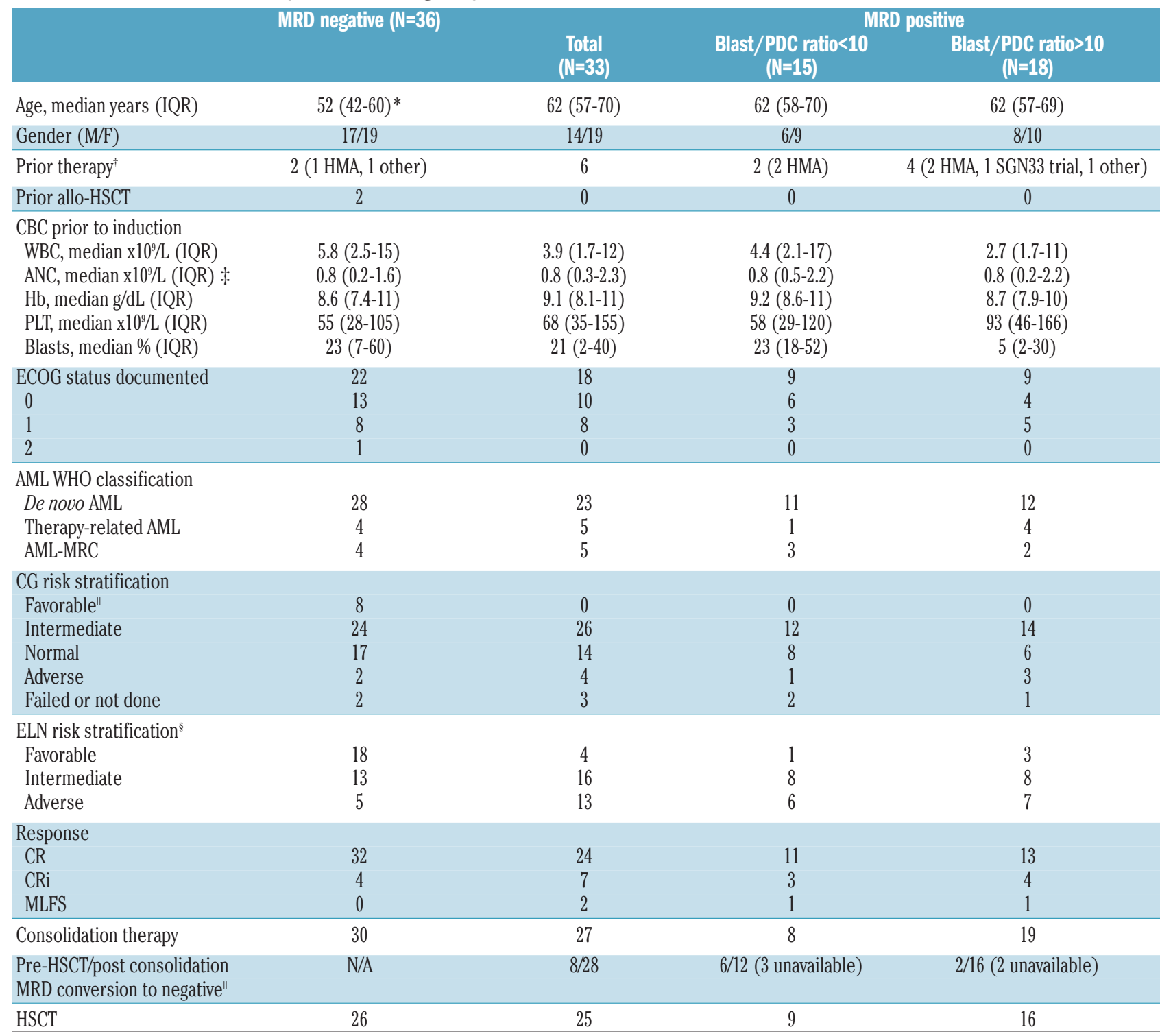

Measurable residual disease (MRD) negative (neg) versus MRD positive (pos), $P=0.01$ MRD neg versus Blast/plasmacytoid dendritic cell (PDC) ratio $<10$. $P=0.005$ (Fisher exact) MRD neg versus MRD pos. ${ }^{5} P=0.0008$ (Fisher exact) MRD neg versus MRD pos. $P=0.02$ (Fisher exact) Blast/PDC ratio $<10$ versus Blast/PDC ratio $>10$. ANC: absolute neutrophil count; Hb: hemoglobin; PLT: platelets; AML-MRC: AML with myelodysplasia-related changes; CG: cytogenetic; CR: complete remission; CRi: complete remission with incomplete counts recovery; MLFS: marrow leukemia free state. PDC: plasmacytoid dendritic cells; N: number; M: male; F: female; IQR: Interquartile Range; allo-HSCT: allogeneic hematopoietic stem cell transplantation; CBC: complete blood cell;WBC: white blood cell; ECOG: Eastern Cooperative Oncology Group;WHO:World Health Organization. 
Supplementary Table S3). The numbers of patients in the two groups were too low for statistical significance.

We further investigated if the MRD-pos patients with blast/PDC ratio $<10$ would have a higher rate of $M R D$ clearance at a later post induction time point (post consolidation and/or pre-HSCT). Excluding five patients ( 3 with a blast/PDC ratio $<10$ and 2 with a ratio $>10$ ) who did not have a follow-up MRD test, MRD-pos patients with blast/PDC ratio $<10$ had a four times higher MRD clearance rate than MRD-pos patients with a ratio $>10$ ( 6 of 12 , $50 \%$ vs. 2 of $16,12.5 \%$; Fisher exact test, $P=0.04$ ).

\section{Restoration of normal hematopoiesis in measurable residual disease-pos patients with blast/plasmacytoid dendritic cell ratio $<10$}

To understand why the rate of MRD clearance was higher in the MRD-pos patients with blast/PDC ratio $<10$ (vs. >10), we reviewed the clinical characteristics and flow cytometric immunophenotype of these patients (Table 1 and Online Supplementary Table S4). We did not observe a correlation with WHO classification, CG risk, ELN risk and the rate of achieving $\mathrm{CR}$, in accordance with prior observations of MRD positivity being the dominant predictive factor post induction. ${ }^{9}$ In patients with a blast/PDC ratio $>10$, along with marked reduction of $\mathrm{PDC}$, regenerating HSCs (CD34+ cells) with normal immunophenotype were not seen (Figure 4A-D). In contrast, prominent PDC populations were present in all patients with a blast/PDC ratio $<10$ (Figure 4E-H). Moreover, 13 out of these 15 patients showed regenerating HSC with normal immunophenotype in addition to a distinct abnormal myeloid blast population, indicating detectable normal hematopoietic regeneration alongside low-level persistent disease in these MRD-pos patients with normalized blast/PDC ratios (Online Supplementary Table S4).

\section{Blast/plasmacytoid dendritic cell ratio $>10$ at late} time points is associated with poor outcome

We examined the dynamics of blast/PDC ratio at later time points (post consolidation and/or pre-transplant): all the post-induction MRD-neg patients remained MRD-neg and the blast/PDC ratio remained $<10$. Among postinduction MRD-pos patients, 15 had post induction blast/PDC ratio $<10$ : 13 remained $<10(2$ died $)$ and two patients converted $>10$ (both died). Eighteen patients had post induction blast/PDC ratio $>10$ : nine remained $>10(7$ died) and nine converted to $<10$ (none died). Although it is challenging to compare RFS and OS because of the relatively low number of subjects, these results suggest that patients with post induction blast/PDC ratio of $>10$ can convert to ratio $<10$, and these converted patients have a favorable outcome. Patients with post induction blast/PDC ratio $<10$ rarely convert to ratio $>10$ and if it does occur, these patients appear to have poor outcome.

We next evaluated if blast/PDC ratio can further risk stratify MRD-pos patients at late time points (prior to HSCT). Only patients receiving HSCT were included and the date of transplantation was chosen as a unifying starting point for outcome. Patients with relapsed disease ( $\geq 5 \%$ blasts by morphology) prior to HSCT were excluded. We had $32 \mathrm{MRD}$-neg patients (all had blast/PDC ratio $<10)$ and $16 \mathrm{MRD}$-pos patients (10 had blast/PDC ratio

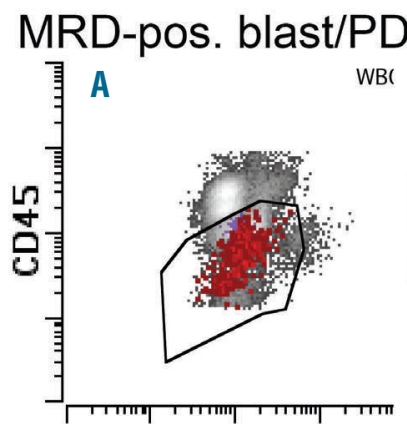

SSC-H

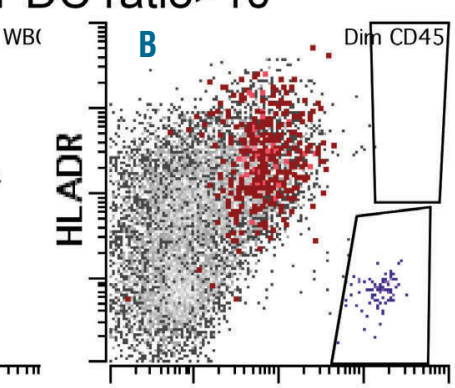

CD123

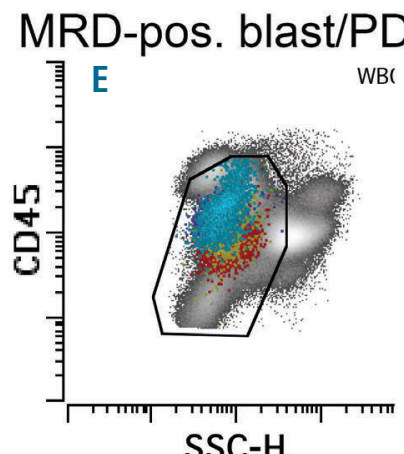

SSC-H
WBI

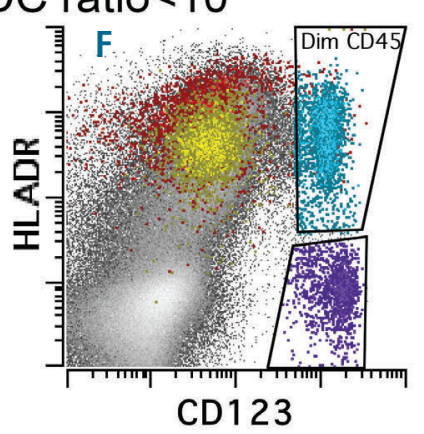

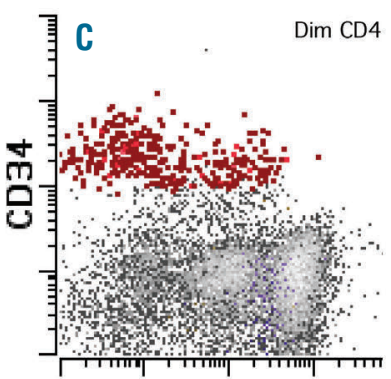

CD11b

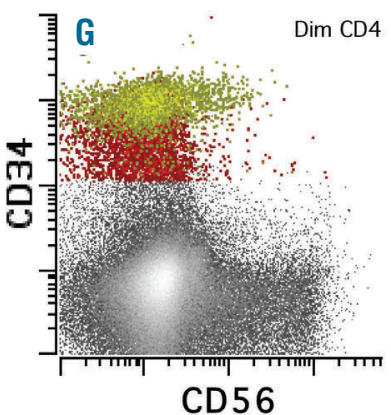

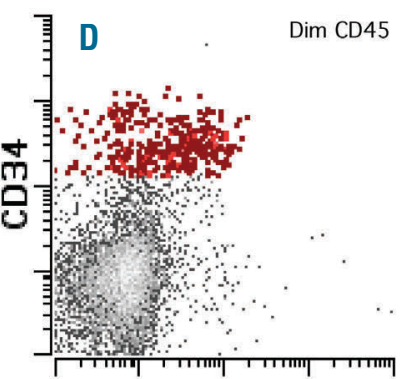

CD25

Figure 4. Examples of abnormal blast immunophenotype in measurable residual disease (MRD)-pos patients with blast/plasmacytoid dendritic cell (PDC) ratio $>10$ (A-D) versus <10 (E-H). (A-D) PDC are markedly decreased (B) and the blasts have abnormal expression of CD11b (partial, C) and CD25 (D). (E-H) PDC are well preserved (F) and a subset of the blasts (highlighted in yellow) showed abnormal expression of CD38 (absent, data not shown), CD56 (G), and CD25 (H). Red: CD34positive blasts; blue: PDC; purple: basophils; yellow: abnormal blasts gated on CD34-positive and CD38-negative expression (gates not shown). 
$<10$ and 6 had ratio $>10$ ). MRD-neg patients had a superior RFS and OS. MRD-pos patients with blast/PDC $<10$ had comparable or slightly inferior RFS and OS (Figure 5A and $\mathrm{B}$ ). Although the number was small, the analysis suggested that MRD-pos patients with blast/PDC ratio $>10$ had significantly worse RFS and OS.

\section{Discussion}

Measurable residual disease is an independent prognostic indicator in AML that is important for risk stratification and for planning future treatment. ${ }^{1,2,4}$ However, the major obstacles in interpretation and standardization of the methodology of monitoring MRD by flow cytometry has meant that this has only gradually been applied to risk stratification in AML. ${ }^{4,26,28}$ LAIP-based DfN approaches are qualitative in principle, highly expertise-dependent, and subject to errors of interpretation. ${ }^{4}$ An objective, quantitative flow cytometry-based approach to identify MRD in AML has yet to be developed. Our study is the first attempt of its kind to establish such an objective and simple approach. We demonstrate that PDC proportion can be reliably quantified and that a blast/PDC ratio $>10$ has very high specificity and moderate sensitivity for detecting MRD positivity. More importantly, blast/PDC ratio following induction chemotherapy has a strong predictive value for relapse, OS and RFS, comparable to DfN MFCbased identification of AML MRD in a uniformly treated $\mathrm{AML}$ cohort. Moreover, blast/PDC ratios at late time points, i.e. post consolidation and/or pre-transplant, can also help risk stratification of MRD-pos patients. While we investigated the most common therapy regimen, the study was confined to a single large academic institution. We attempted to evaluate another cohort of AML patients treated with hypomethylating agents, but these patients rarely enter remission or experience PDC recovery, making evaluation impossible (data not shown). Nonetheless, the measurement of blast/PDC ratio shows promise for AML risk stratification. This method does not require identification of MRD through specific recognition of DfN myeloid blasts, but rather allows for the calculation of the ratio of total myeloid blasts to PDC. Owing to its objective calculation and simple methodology, potentially, this approach can be widely applied in various laboratories with different levels of expertise. Our results should also encourage investigation into other objective and quantitative parameters in flow cytometry-based MRD evaluation, in a similar way to how the Ogata scoring system was developed for the diagnosis of myelodysplastic syndrome. ${ }^{43-45}$ In this regard, the proportions of other populations including immature progenitors warrant further investigation in AML.

Although our results are limited by small numbers, the suggestion of a higher MRD clearance rate in patients who were post-induction MRD-pos but had a blast/PDC ratio $<10$ is intriguing. The DfN approach-based MRD positivity in all cases was confirmed by a second review arguing against diagnostic errors. Among post-induction MRDpos patients with blast/PDC ratio <10 (vs. >10), a larger proportion was converted to MRD negativity and a lower incidence of relapse was observed. Several potential mechanisms may explain this finding. DfN-based MRD positive blasts in AML patients with blast/PDC ratio $<10$ may have lost leukemic potential, whereby damage to residual leukemic blasts led to delayed cell death. Additionally, leukemic blasts in marrows with a blast/PDC ratio $<10$ may have been cleared through therapeutic selection or differentiation with consolidation therapy. Restoration of PDC might suggest an inherent chemotherapy sensitivity and progression towards normal hematopoiesis in which remaining blasts are susceptible
A

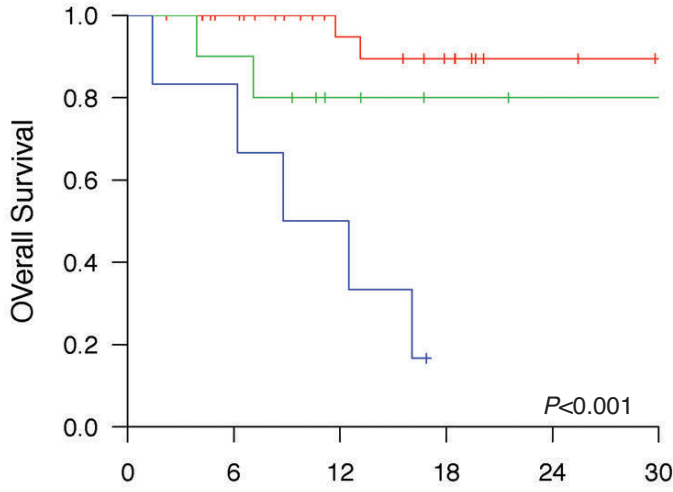

Months from HSCT

\section{No. at risk}

MRD-/Ratio $<10 \quad 32$

MRD $+/$ Ratio $<10 \quad 10$

MRD+/Ratio $>10 \quad 6$

$\begin{array}{ccccc}27 & 18 & 14 & 9 & 7 \\ 9 & 5 & 3 & 2 & 2 \\ 5 & 3 & 0 & 0 & 0\end{array}$

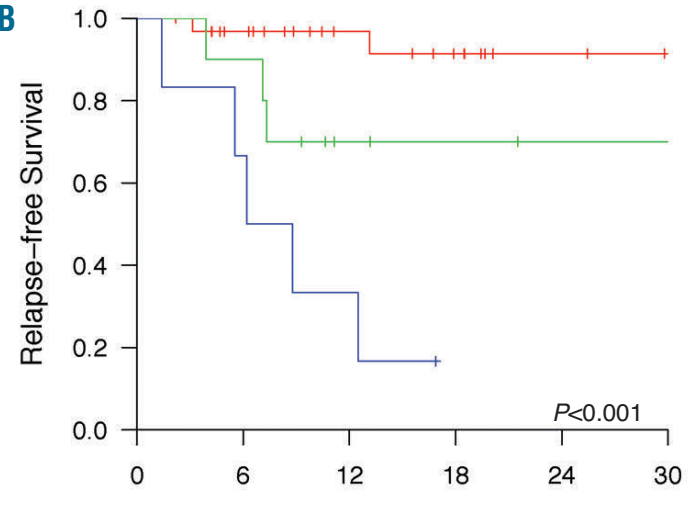

Months from HSCT

No. at risk

MRD-/Ratio $<10 \quad 32$ MRD $+/$ Ratio $<10 \quad 10$ MRD $+/$ Ratio $>10 \quad 6$

\section{6}

9
18

4
2

$\begin{array}{ccc}14 & 9 & 7 \\ 3 & 2 & 2 \\ 0 & 0 & 0\end{array}$

Figure 5. Kaplan-Meier survival analysis based on pre-hematopoietic stem cell transplantation (HSCT) measurable residual disease (MRD) status and blast/plasmacytoid dendritic cell ratio (PDC). (A) Overall survival (OS) of MRD-negative (neg), MRD-positive (pos) with blast/PDC ratio <10, and MRD-pos with ratio >10 groups. (B) Relapse-free survival (RFS) of MRD-neg, MRD-pos with blast/PDC ratio <10, and MRD-pos with ratio >10 groups. 
to further reduction in residual disease from remaining therapy. ${ }^{1}$ Finally, restoration of PDC might also reflect the emergence of normal host mechanisms, such as immune surveillance controlling low-level MRD. Consistent with the latter possibility, we observed regenerating normal hematopoiesis in patients with MRD positivity but a blast/PDC ratio $<10$, which may play a critical role in clearing residual leukemic blasts. Regardless of the mechanisms underlying this association, blast/PDC ratio $<10$ provides additional value in identifying this particular group of potentially lower-risk MRD positive patients at very early time points, which has not been possible with current $\mathrm{MRD}$ detection methods. Identifying this subset of patients may benefit critical clinical decision-making with regards to potential MRD-targeted therapy or allogeneic HSCT. The relatively favorable outcome of this subset of patients (MRD-pos with ratio <10) might also explain the powerful predictive value of blast/PDC ratio on OS and RFS as compared to MRD status, despite the imperfect correlation between blast/PDC ratio and MRD status in this particular subset of patients.

The benefit of PDC recovery on outcome also extends to late time points. In fact, $\mathrm{PDC}$ recovery mitigates $\mathrm{MRD}$ positivity and predicts better outcome, suggesting that low-level MRD in the presence of normal hematopoiesis recovery portends a larger benefit of HSCT. On the other hand, based on a limited number of patients, our study suggests that lack of PDC recovery prior to HSCT is associated with extremely poor outcome. If validated by other studies, this would question the benefit of HSCT prior to PDC restoration in this particular subset of patients. Further studies are needed to investigate if these patients would benefit from additional therapies that facilitate PDC recovery.

Plasmacytoid dendritic cell differentiation is regulated by multiple transcription factors including GATA-2, IKAROS, IRF8, TCF4 and ID2. ${ }^{46-48}$ Although higher levels of peripheral blood PDC was suggested in patients with FLT3-ITD ${ }^{+} \mathrm{AML}^{33}$ we did not observe this correlation (data not shown). What mutations in AML regulate PDC differentiation is completely unknown. The decreased PDC differentiation in AML is likely related to overall maturation arrest observed in leukemic blasts. In MRDpos patients with blast/PDC ratio $<10$, there were often two distinct blast populations: one clearly abnormal and the other likely regenerating normal blasts. In this situation, it is reasonable to speculate that the PDC are differentiated from normal blasts rather than leukemic blasts. The significance of blocked PDC differentiation has not been well studied in AML. A few studies suggest low-level reconstitution of PDC post-HSCT in AML is associated with higher relapse rate. ${ }^{49-51}$ It would be interesting to know the MRD status in those patients. As PDC are the major cell type producing type I interferon, the immune regulatory role of $\mathrm{PDC}$ in AML also warrants further investigation.

The caveats of this retrospective study include the problems of a single-center study and the relatively small number of patients, which might not have the power to identify moderate but clinically significant differences in survival outcomes. In spite of this, using the blast/PDC ratio, we provide the first objective and quantitative MFC method to risk stratify AML. Increased blast/PDC ratio correlates with residual leukemia, is highly specific for MRD positivity in post-induction patients, and strongly predicts poorer OS and higher likelihood of relapse. Normal PDC proportion predicts MRD clearance in postinduction MRD-pos patients, and is associated with restoration of normal hematopoiesis. Pre-HSCT blast/PDC ratio in combination with MRD status serves as a powerful predictor of post-HSCT outcomes. We propose that using the blast/PDC ratio will facilitate standardization of MFC-based MRD approaches and corroborate MRD to risk stratify AML.

\section{Acknowledgments}

This study was funded by the Center for Hematologic Malignancies at MSKCC and in part through the NIH/NCI Cancer Center Support Grant P30 CA008748. WX is supported by a startup fund from Department of Pathology at the Memorial Sloan Kettering Cancer Center. ADG is supported by a Young Investigator Award from the Conquer Cancer Foundation of the American Society of Clinical Oncology and a Scholar Award from the American Society of Hematology (ASH). MBG is supported by the Lymphoma Research Foundation and a Scholar Award from ASH. A.D.V. is supported by a National Cancer Institute career development grant K08 CA215317 and an EvansMDS Young Investigator grant from the Edward P. Evans Foundation.

\section{Reference}

1. Chen X, Wood BL. Monitoring minimal residual disease in acute leukemia: Technical challenges and interpretive complexities. Blood Rev. 2017;31(2):63-75.

2. Bloomfield CD, Estey E, Pleyer L, et al. Time to repeal and replace response criteria for acute myeloid leukemia? Blood Rev. 2018;32(5):416-425.

3. Ossenkoppele G, Schuurhuis GJ. MRD in AML: does it already guide therapy decision-making? Hematology American Society of Hematology Education Program. 2016;2016(1):356-365.

4. Schuurhuis GJ, Heuser M, Freeman S, et al. Minimal/measurable residual disease in AML: a consensus document from the European LeukemiaNet MRD Working Party. Blood. 2018;131(12):1275-1291.

5. Freeman SD, Virgo P, Couzens $S$, et al.
Prognostic relevance of treatment response measured by flow cytometric residual disease detection in older patients with acute myeloid leukemia. J Clin Oncol. 2013:31(32):4123-4131.

6. Terwijn M, van Putten WL, Kelder A, et al. High prognostic impact of flow cytometric minimal residual disease detection in acute myeloid leukemia: data from the HOVON/SAKK AML 42A study. J Clin Oncol. 2013;31(31):3889-3897.

7. Rubnitz JE, Inaba H, Dahl G, et al. Minimal residual disease-directed therapy for childhood acute myeloid leukaemia: results of the AML02 multicentre trial. Lancet Oncol. 2010;11(6):543-552.

8. San Miguel JF, Vidriales MB, Lopez-Berges C, et al. Early immunophenotypical evaluation of minimal residual disease in acute myeloid leukemia identifies different patient risk groups and may contribute to postinduction treatment stratification. Blood. 2001;98(6):1746-1751

9. Chen X, Xie H, Wood BL, Walter RB, et al. Relation of clinical response and minimal residual disease and their prognostic impact on outcome in acute myeloid leukemia. Clin Oncol. 2015;33(11):1258-1264.

10. Othus M, Wood BL, Stirewalt DL, et al Effect of measurable ('minimal') residual disease (MRD) information on prediction of relapse and survival in adult acute myeloid leukemia. Leukemia. 2016;30(10):20802083.

11. Venditti A, Buccisano F, Del Poeta G, et al. Level of minimal residual disease after consolidation therapy predicts outcome in acute myeloid leukemia. Blood. 2000;96(12):39483952.

12. Kern W, Voskova D, Schoch C, Hiddemann W, Schnittger S, Haferlach T. Determination of relapse risk based on assessment of mini- 
mal residual disease during complete remission by multiparameter flow cytometry in unselected patients with acute myeloid leukemia. Blood. 2004;104(10):3078-3085

13. Buccisano F, Maurillo L, Spagnoli A, et al. Cytogenetic and molecular diagnostic characterization combined to postconsolidation minimal residual disease assessment by flow cytometry improves risk stratification in adult acute myeloid leukemia. Blood. 2010;116(13):2295-2303.

14. San Miguel JF, Martinez A, Macedo A, et al. Immunophenotyping investigation of minimal residual disease is a useful approach for predicting relapse in acute myeloid leukemia patients. Blood. 1997;90(6):2465-2470.

15. Buccisano F, Maurillo L, Gattei V, et al. The kinetics of reduction of minimal residual disease impacts on duration of response and survival of patients with acute myeloid leukemia. Leukemia. 2006;20(10):17831789.

16. Al-Mawali A, Gillis D, Hissaria P, Lewis I. Incidence, sensitivity, and specificity of leukemia-associated phenotypes in acute myeloid leukemia using specific five-color multiparameter flow cytometry. Am J Clin Pathol. 2008;129(6):934-945.

17. van der Velden VHJ, van der Sluijs-Geling A, Gibson BES, et al. Clinical significance of flowcytometric minimal residual disease detection in pediatric acute myeloid leukemia patients treated according to the DCOG ANLL97/MRC AML12 protocol. Leukemia. 2010;24(9):1599-1606.

18. Walter RB, Gooley TA, Wood BL, et al. Impact of pretransplantation minimal residual disease, as detected by multiparametric flow cytometry, on outcome of myeloablative hematopoietic cell transplantation for acute myeloid leukemia. J Clin Oncol. 2011:29(9):1190-1197.

19. Walter RB, Buckley SA, Pagel JM, et al. Significance of minimal residual disease before myeloablative allogeneic hematopoietic cell transplantation for AML in first and second complete remission. Blood. 2013;122(10):1813-1821.

20. Araki D, Wood BL, Othus M, et al. Allogeneic Hematopoietic Cell Transplantation for Acute Myeloid Leukemia: Time to Move Toward a Minimal Residual Disease-Based Definition of Complete Remission? J Clin Oncol. 2016;34(4):329-336.

21. Walter RB, Gyurkocza B, Storer BE, et al. Comparison of minimal residual disease as outcome predictor for AML patients in first complete remission undergoing myeloablative or nonmyeloablative allogeneic hematopoietic cell transplantation. Leukemia. 2015;29(1):137-1344.

22. Dohner $\mathrm{H}$, Estey E, Grimwade $\mathrm{D}$, et al. Diagnosis and management of AML in adults: 2017 ELN recommendations from an international expert panel. Blood. 2017;129(4):424-447.

23. Freeman SD, Hills RK, Virgo $P$, et al. Measurable Residual Disease at Induction Redefines Partial Response in Acute Myeloid Leukemia and Stratifies Outcomes in Patients at Standard Risk Without NPM1 Mutations. J Clin Oncol. 2018;36(15):14861497.
24. Langebrake C, Creutzig U, Dworzak M, et al. Residual disease monitoring in childhood acute myeloid leukemia by multiparameter flow cytometry: the MRD-AML-BFM Study Group. J Clin Oncol. 2006;24(22):3686-3692.

25. Baer MR, Stewart CC, Dodge RK, et al. High frequency of immunophenotype changes in acute myeloid leukemia at relapse: implications for residual disease detection (Cance and Leukemia Group B Study 8361). Blood. 2001;97(11):3574-3580.

26. Wood BL, Arroz M, Barnett D, et al. 2006 Bethesda International Consensus recommendations on the immunophenotypic analysis of hematolymphoid neoplasia by flow cytometry: optimal reagents and reporting for the flow cytometric diagnosis of hematopoietic neoplasia. Cytometry B Clin Cytom. 2007;72 Suppl 1:S14-22

27. Kalina T, Flores-Montero J, van der Velden $\mathrm{VH}$, et al. EuroFlow standardization of flow cytometer instrument settings and immunophenotyping protocols. Leukemia. 2012;26(9):1986-2010.

28. van Dongen JJ, Lhermitte L, Bottcher S, et al EuroFlow antibody panels for standardized n-dimensional flow cytometric immunophenotyping of normal, reactive and malignant leukocytes. Leukemia. 2012;26(9):1908-1975

29. Reizis B, Bunin A, Ghosh HS, Lewis KL, Sisirak V. Plasmacytoid dendritic cells: recent progress and open questions. Annu Rev Immunol. 2011;29:163-183.

30. Guilliams M, Ginhoux F, Jakubzick C, et al. Dendritic cells, monocytes and macrophages: a unified nomenclature based on ontogeny. Nat Rev Immunol. 2014;14(8):571-578.

31. Merad M, Sathe P, Helft J, Miller J, Mortha A. The dendritic cell lineage: ontogeny and function of dendritic cells and their subsets in the steady state and the inflamed setting. Annu Rev Immunol. 2013;31:563-604.

32. Derolf AR, Laane E, Bjorklund E, Saft L, Bjorkholm M, Porwit A. Dendritic cells in bone marrow at diagnosis and after chemotherapy in adult patients with acute myeloid leukaemia. Scand J Immunol. 2014;80(6):424-431.

33. Rickmann M, Krauter J, Stamer K, et al. Elevated frequencies of leukemic myeloid and plasmacytoid dendritic cells in acute myeloid leukemia with the FLT3 internal tandem duplication. Ann Hematol. 2011;90(9):1047-1058.

34. Rovati B, Mariucci S, Manzoni M, Bencardino K, Danova M. Flow cytometric detection of circulating dendritic cells in healthy subjects. Eur J Histochem. 2008;52(1):45-52.

35. Hsu AP, Johnson KD, Falcone EL, et al. GATA2 haploinsufficiency caused by mutations in a conserved intronic element leads to MonoMAC syndrome. Blood. 2013;121:3830-7, S1-7.

36. Hsu AP, Sampaio EP, Khan J, et al. Mutations in GATA2 are associated with the autosomal dominant and sporadic monocytopenia and mycobacterial infection (MonoMAC) syndrome. Blood. 2011;118(10):2653-2655.

37. Naresh KN, Pavlu J. Plasmacytoid dendritic cell nodules in bone marrow biopsies of chronic myelomonocytic leukemia. Am J
Hematol. 2010;85(11):893.

38. Song HL, Huang WY, Chen YP, Chang KC Tumorous proliferations of plasmacytoid dendritic cells and Langerhans cells associated with acute myeloid leukaemia. Histopathology. 2012;61(5):974-983.

39. Vuckovic S, Fearnley DB, Gunningham S, Spearing RL, Patton WN, Hart DN Dendritic cells in chronic myelomonocytic leukaemia. Br J Haematol. 1999;105(4):974 985.

40. Cheng DT, Cheng I, Mitchell TN, et al Detection of mutations in myeloid malignancies through paired-sample analysis of microdroplet-PCR deep sequencing data. J Mol Diagn. 2014;16(5):504-518.

41. Getta BM, Devlin SM, Levine RL, et al Multicolor Flow Cytometry and Multi-Gene Next Generation Sequencing are Complementary and Highly Predictive for Relapse in Acute Myeloid Leukemia Following Allogeneic Transplant. Bio Blood Marrow Transplant. 2017;23(7):1064-1071

42. Uno $H$, Cai T, Pencina MJ, D'Agostino RB, Wei LJ. On the C-statistics for evaluating overall adequacy of risk prediction procedures with censored survival data. Stat Med. 2011;30(10):1105-1117.

43. Westers TM, Cremers EM, Oelschlaegel U et al. Immunophenotypic analysis of erythroid dysplasia in myelodysplastic syndromes. A report from the IMDSFlow working group. Haematologica. 2017;102(2):308319.

44. Della Porta MG, Picone C, Pascutto C, et al Multicenter validation of a reproducible flow cytometric score for the diagnosis of low-grade myelodysplastic syndromes: results of a European LeukemiaNET study. Haematologica. 2012;97(8):1209-1217.

45. Ogata K, Della Porta MG, Malcovati L, et al Diagnostic utility of flow cytometry in lowgrade myelodysplastic syndromes: a prospective validation study. Haematologica. 2009;94(8):1066-1074

46. Swiecki M, Colonna M. The multifaceted biology of plasmacytoid dendritic cells. Nat Rev Immunol. 2015;15(8):471-485.

47. Allman D, Dalod M, Asselin-Paturel C, et al Ikaros is required for plasmacytoid dendritic cell differentiation. Blood. 2006;108(13): 4025-4034.

48. Tassone L, Moratto D, Vermi W, et al Defect of plasmacytoid dendritic cells in warts, hypogammaglobulinemia, infections, myelokathexis (WHIM) syndrome patients. Blood. 2010;116(23):4870-4873

49. Auletta JJ, Devine SM, Waller EK Plasmacytoid dendritic cells in allogeneic hematopoietic cell transplantation: benefit or burden? Bone Marrow Transplant. 2016;51(3):333-343

50. Elze MC, Ciocarlie $O$, Heinze $A$, et al. Dendritic cell reconstitution is associated with relapse-free survival and acute GVHD severity in children after allogeneic stem cell transplantation. Bone Marrow Transplant. 2015;50(2):266-273.

51. Su RJ, Green R, Chen M. Enumeration of bone marrow plasmacytoid dendritic cells by multiparameter flow cytometry as a prognostic marker following allogeneic hematopoietic stem cell transplantation. Blood Cells Mol Dis. 2018;69:107-112. 